\title{
Histiocytose langerhansienne chez un bébé de 5 mois
}

\author{
Neil Chanchlani MBChB MSc, Simon C. Parke MBChB, James W. Hart MBBS
}

- Citation : CMAJ 2021 January 4;193:E23. doi : 10.1503/cmaj.201151-f

Voir la version anglaise de l'article ici : www.cmaj.ca/lookup/doi/10.1503/cmaj.201151

$\mathbf{U}$

n garçon de 2 mois a été évalué par son médecin de famille en raison de la présence de pétéchies, d'érythème confluent, de pustules croûteuses et d'une hyperémie dans les plis cutanés cervicaux, axillaires et inguinaux ainsi que les plis cutanés situés derrière les oreilles. Le médecin a posé un diagnostic d'eczéma infectieux et a prescrit du dipropionate de bétaméthasone topique et de l'amoxicilline à prise orale, mais aucune amélioration n'a été observée.

À l'âge de 5 mois, le garçon, qui suivait bien sa courbe de croissance, a été emmené à l'hôpital. Ses problèmes cutanés s'étaient aggravés (figure 1), et il avait de la fièvre, des vomissements biliaires, de la diarrhée et de l'œdème depuis 2 jours. À l'analyse sanguine, son taux d'hémoglobine était de $84 \mathrm{~g} / \mathrm{L}$ (plage normale 95-135 g/L), son volume globulaire moyen, de $86 \mathrm{fL}$ (plage normale $74-108 \mathrm{fL}$ ), sa numération leucocytaire, de $11,4 \times 10^{9} / \mathrm{L}$ (plage normale $6,0-$ $\left.17,5 \times 10^{9} / \mathrm{L}\right)$, sa numération plaquettaire, de $107 \times 10^{9} / \mathrm{L}$ (plage normale $150-400 \times 10^{9} / \mathrm{L}$ ), son taux de bilirubine, de $9 \mu \mathrm{mol} / \mathrm{L}$ (valeur normale $<21 \mu \mathrm{mol} / \mathrm{L}$ ), et son taux d'albumine, de $17 \mathrm{~g} / \mathrm{L}$ (plage normale $30-45 \mathrm{~g} / \mathrm{L}$ ). Ses fonctions rénales et hépatiques étaient normales. Une lésion lytique à l'extrémité proximale du fémur gauche a été découverte à l'examen du squelette.

Les biopsies cutanées et duodénales ont révélé une histiocytose langerhansienne, et l'ensemble de l'investigation a montré que celleci touchait plusieurs systèmes (cutané, sanguin, osseux et gastrointestinal). L'enfant a peu répondu

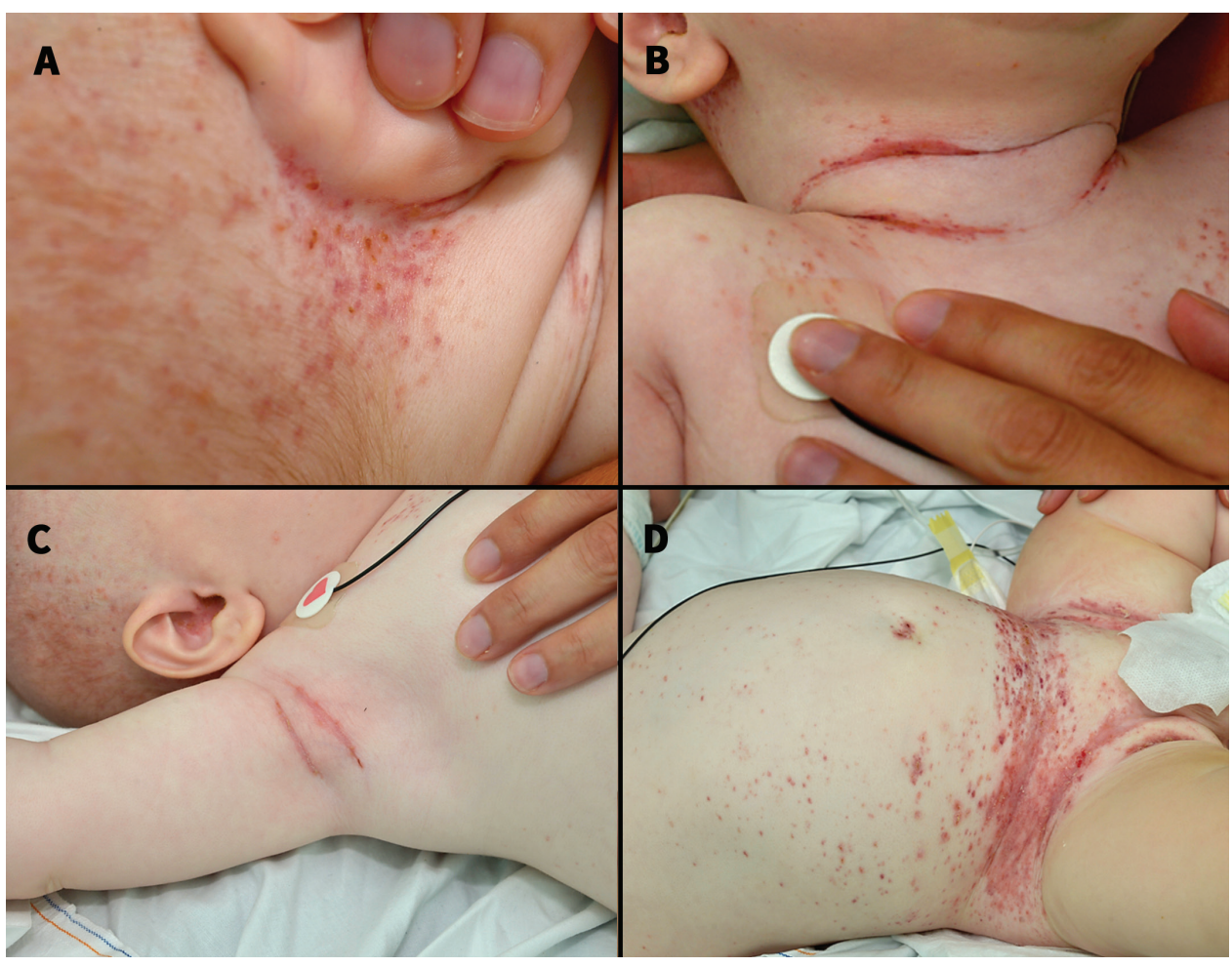

Figure 1 : Photographies d'un garçon de 5 mois présentant des pétéchies, un érythème confluent, des papules croûteuses et une hyperémie dans les plis cutanés A) des oreilles; B) du cou; C) des aisselles; et D) de l'aine. On observe également des vésicules et des ulcères dans les plis des oreilles et de l'aine, ainsi que des zones macérées dans le cou, aux aisselles et à l'aine. 
une polyurie et une polydipsie dues à un diabète insipide, des otites moyennes chroniques et des symptômes d'affection multisystémique, comme une aplasie médullaire (cytopénie), une lymphadénopathie ou une hépatosplénomégalie ${ }^{3}$. Jusqu'à $50 \%$ des personnes atteintes développent des complications ou décèdent ${ }^{4}$.

Parmi les signes indiquant qu'il pourrait s'agir d'autre chose que d'eczéma, notons l'absence de réponse aux stéroïdes topiques, une fièvre d'étiologie inconnue, des pétéchies généralisées non liées à une excoriation, une présentation atypique (p. ex., touchant les aisselles et l'aine) et une irritabilité persistante ${ }^{3}$.

\section{Références}

1. Rigaud C, Barkaoui MA, Thomas C, et al. Langerhans cell histiocytosis: therapeutic strategy and outcome in a 30-year nationwide cohort of 1478 patients under 18 years of age. Br J Haematol 2016;174:887-98.

2. Krooks J, Minkov M, Weatherall AG. Langerhans cell histiocytosis in children: Diagnosis, differential diagnosis, treatment, sequelae, and standardized follow-up. J Am Acad Dermatol 2018;78:1047-56.

3. Haupt R, Minkov M, Astigarraga I, et al. Langerhans cell histiocytosis (LCH): guidelines for diagnosis, clinical work-up, and treatment for patients till the age of 18 years. Pediatr Blood Cancer 2013;60:175-84.

4. Allen CE, Merad M, McClain KL. Langerhans-cell histiocytosis. N Engl J Med 2018;379:856-68
Intérêts concurrents : Neil Chanchlani est titulaire d'une bourse de recherche clinique de Crohn's and Colitis UK. Aucun autre intérêt concurrent n'a été déclaré.

Cet article a été révisé par des pairs.

Les auteurs ont obtenu le consentement des parents.

Affiliation : Royal Devon and Exeter Hospital, Exeter, R.-U.

Propriété intellectuelle du contenu : Il s'agit d'un article en libre accès distribué conformément aux modalités de la licence Creative Commons Attribution (CC BY-NC-ND 4.0), qui permet l'utilisation, la diffusion et la reproduction dans tout médium à la condition que la publication originale soit adéquatement citée, que l'utilisation se fasse à des fins non commerciales (c.-à-d., recherche ou éducation) et qu'aucune modification ni adaptation n'y soit apportée. Voir : https://creativecommons.org/licenses/by-nc-nd/4.0/deed.fr.

Déclaration d'intérêts : Neil Chanchlani est rédacteur associé pour le CMAJ, mais n'a pas participé au processus ayant mené au choix de cet article.

Correspondance : Neil Chanchlani, nchanchlani@doctors.org.uk 